\title{
Support needs and experiences of family members of wounded, injured or sick UK service personnel
}

\author{
Anna Verey, ${ }_{1}^{1}$ M Keeling, ${ }^{2} \mathrm{G}$ Thandi, ${ }^{1}$ S Stevelink, ${ }^{1} \mathrm{~N}$ Fear ${ }^{1}$
}

'Psychological Medicine, King's Centre for Military Health Research, King's College London, London, UK

${ }^{2}$ School of Social Work, Center for Innovation and Research on Veterans and Military Families, University of Southern California, Los Angeles, California, USA

\section{Correspondence to}

Anna Verey, King's Centre for Military Health Research, King's College London, Weston Education Centre, London SE5 9RJ, UK; anna.verey@kcl.ac.uk

Received 29 September 2016 Revised 30 June 2017 Accepted 30 June 2017 Published Online First 9 October 2017
CrossMark

To cite: Verey $A$, Keeling $M$, Thandi G, et al.

$J$ R Army Med Corps

2017:163:388-393.

\section{ABSTRACT}

Introduction When a service person has been wounded, injured or sick (WIS), family members may provide care during their recovery in an unpaid capacity. This may occur in diverse environments including hospitals, inpatient rehabilitation centres, in the community and at home.

Method Thirty-seven family members of WIS personnel were interviewed regarding their support needs, family relationships and use of UK support services. Semistructured, in-depth telephone interviews were used, with data analysis undertaken using a thematic approach.

Results 'Family member involvement' was the main theme under which four subthemes were situated: 'continuity of support', 'proactive signposting and initiating contact', 'psychoeducation and counselling' and 'higher risk groups'. Family members felt they might benefit from direct, consistent and continuous care regardless of the WIS person's injury or engagement type, and whether the WIS person was being treated in a hospital, rehabilitative centre or at home.

Conclusion The findings of this study suggest that family members of WIS personnel value proactive, direct and sustained communication from support service providers. We suggest that families of UK service personnel may benefit from family care coordinators, who could provide continuous and consistent care to family members of WIS personnel.

\section{INTRODUCTION}

The military environment presents the possibility of injuries and fatalities due to arduous training and high-intensity, hostile, combat operations. ${ }^{1}$ Wounded, injured or sick (WIS) personnel may experience injuries attributable to combat operations and non-battle injuries attributable to training, prior injuries, traffic accidents and illnesses. ${ }^{2}$ When a service person (SP) has been wounded, injured or become sick, family members may provide care during their recovery in an unpaid capacity and in diverse environments including hospitals, inpatient rehabilitation centres, in the community and at home; under such circumstances, family members can be considered informal caregivers. ${ }^{3}$

Caregiver 'burden' is a term used to describe the negative consequences experienced by a caregiver. ${ }^{4}$ Research has demonstrated that if a caregiver is well supported, then the recovery of the care recipient can improve; otherwise, caregivers may have less capacity to provide care to their family member(s) and also themselves be at risk of physical and mental health consequences. ${ }^{5}$

The UK Ministry of Defence (MoD) is committed to ensuring that the families of WIS personnel are

\section{Key messages}

Family members would value proactive, direct and sustained communication with support service providers (where this does not compromise the wishes of the wounded, injured or sick (WIS) person).

- Family members of WIS service personnel may benefit from 'family care coordinators' who would effectively signpost, increase family involvement and provide continuous and consistent care.

- Empirical investigation warranted to examine whether systematic differences occur between support service provision for family members of WIS personnel with operational or non-operational injuries.

properly supported, as set out in the Armed Forces Covenant. ${ }^{6}$ The MoD states that the responsibility for the welfare of service personnel and their families is 'incumbent upon' service personnel themselves and that service personnel are expected to be responsible for maintaining the communication between their families and support service provision. ${ }^{7}$

Concerns have been raised and outlined in a House of Commons Defence Committee report and raised in the Armed Forces Covenant report ${ }^{68}$ about the care and support available to families of WIS service personnel. Furthermore, prior research has focused on caregiving in a civilian rather than military context.

The aim of this study was to examine the changing needs and experiences of family members of WIS personnel in the UK, in order to provide evidence relating to support provided by the MoD, Other Governmental Departments and service/ civilian charities, so as to identify best practice and inform future policy.

\section{METHOD \\ Participants}

A total of 500 WIS personnel were identified via the Wounded, Injured and Sick Management Information System. This is the database holding information of WIS service personnel who have been off work due to health problems for more than 7 days. In order to achieve a representative sample of family members, WIS personnel were stratified by engagement type (regular or reserve), service (Royal Air Force (RAF), Navy or Army) and operational and non-operational injury/illness (an operational injury/illness is deployment related 


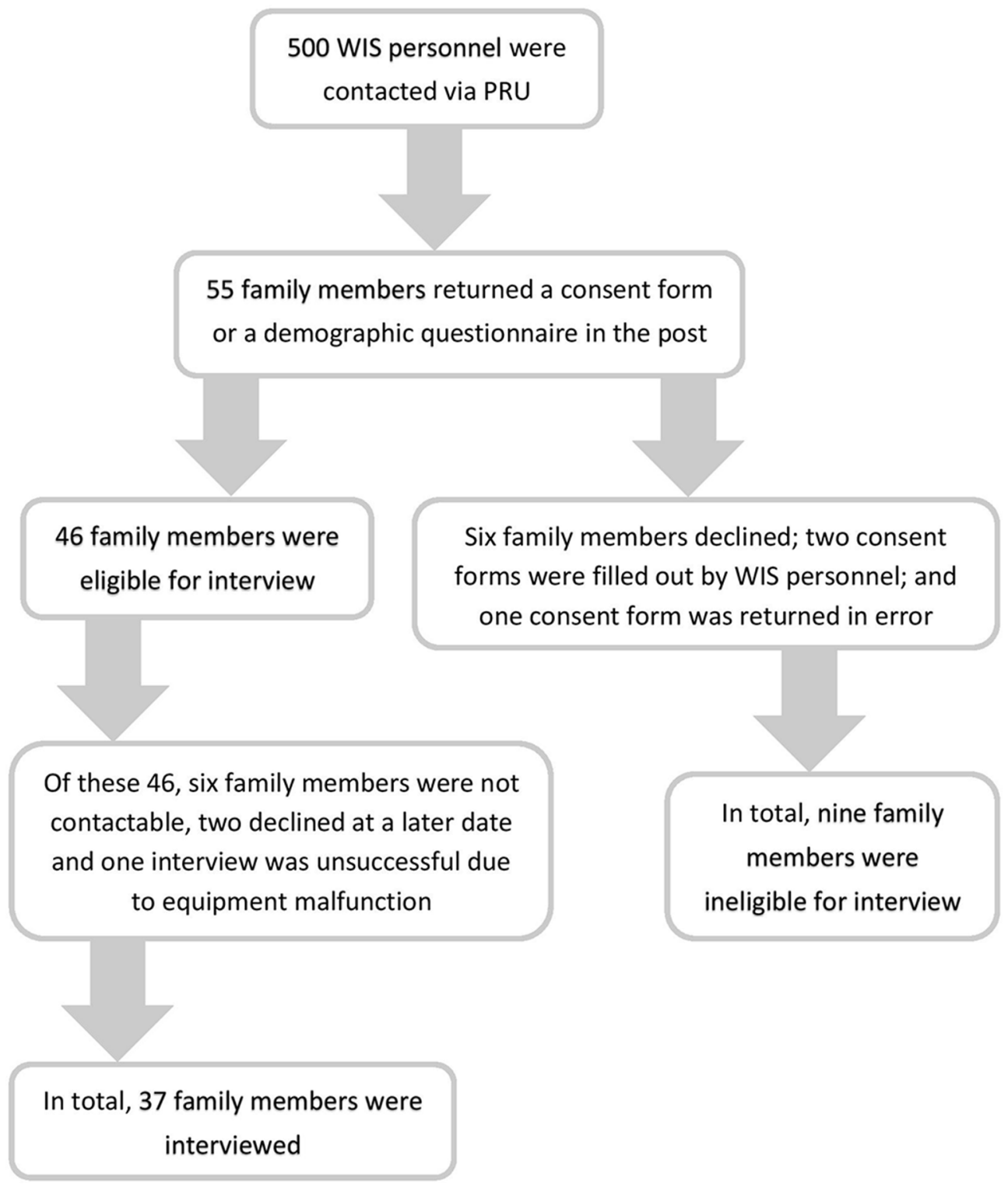

Figure 1 Recruitment flow diagram. PRU, Personnel Recovery Unit; WIS, wounded, injured or sick.

and a non-operational injury/illness is non-deployment related) (online supplementary table 1 ).

\section{Procedure}

A total of 500 invitation packs were posted to individual WIS personnel at their respective Personnel Recovery Units (PRUs). A PRU is a military unit that takes command of the care of WIS personnel while they are recovering. Each invitation pack contained a letter to the WIS person inviting them to pass the pack on to a family member of their choice. Family members were eligible to take part if they were more than 18 years and were parents, partners, spouses or siblings. On receipt of any completed consent forms from eligible participants, the study team made contact with the family member to arrange a time to conduct a telephone interview (Figure 1).

\section{Data collection}

The semistructured interview schedule (online supplementary table 2) was informed by themes from the literature ${ }^{9}$ and developed by leading members of the research team and departmental colleagues with expertise in conducting qualitative research, to address questions of family members' use of support services, possible support needs and the impact of the WIS person's illness/injury.

Participant interviews were carried out between March and August 2014. At the start of each interview, the purpose of the research was reiterated, and consent was retaken. Each interview took approximately 1 hour to complete, and no repeat interviews were carried out. Dictaphones were used to record interviews; field notes were collected after each interview.

\section{Analysis}

All interviews were transcribed including all spoken words; furthermore, laughter, sighs, significant pauses and hesitations were noted. Participants and WIS personnel were allocated pseudonyms.

Data were analysed using thematic analysis, a qualitative data analysis technique that facilitates the identification, analysis and reporting of themes within a data set. ${ }^{10} \mathrm{~A}$ detailed, inductive analysis of data occurred, such that data were coded without trying to match them to any pre-existing coding frameworks or the researchers' preconceptions. ${ }^{10}$ 
Analysis began with familiarisation of interview data. Researchers listened to the recording of interviews twice, after which they read and reread the transcripts and started to make notes of emerging codes (all subsequent analysis was conducted with the assistance of NVivo10). Each transcript was subject to line-by-line analysis which led to the development of initial codes; this process was repeated until initial codes were developed, leading to the development of subthemes. Spider diagrams of possible master themes and subthemes were produced, and the remaining transcripts analysed, creating an initial coding framework. Subsequent meetings between the two researchers and additional members of the research team took place to discuss further the emergent subthemes and any new codes. In these meetings, the coding framework was updated, and analysis continued until all transcripts had been analysed and the subthemes for each master theme had been agreed. The structure of the thematic framework which developed as a result of the analysis process was updated throughout the analysis (the term analysis refers to and includes the writing process). Saturation was reached within the analysis process, meaning that no new codes, subthemes or master themes were identified.

During analysis, the two researchers cross-coded three transcripts, that is, coded a random selection of transcripts to check the reliability of the analysis and to discuss how the subthemes may be related to each other. To further ascertain the reliability of the analysis, an experienced colleague, who was blind to the study aims, read, coded and shared their emerging codes from three randomly selected transcripts. Given the high degrees of similarity between the emerging codes and cross-coded transcripts, no major adjustments to the thematic framework were made.

\section{Ethical considerations}

Ethical approval was sought and received from the UK Ministry of Defence Research Ethics Committee (502MOD13). A clinical advisor provided clinical risk assessment training to the research team and was available throughout the study to provide clinical call backs to the participants, if they were distressed, suicidal or asking for help. None of the participants were deemed to be experiencing levels of clinical risk; therefore, none were referred to the clinical advisor.

\section{RESULTS}

Out of the 500 WIS personnel contacted, 37 eligible family members were interviewed. Of these 37 family members, there were 22 spouses/husbands, three partners, eight mothers and four fathers of WIS personnel (Table 1). WIS personnel were deemed to have operational, non-operational or 'uncertain' injuries and had served in all three services. An injury was deemed 'uncertain' by the study team when the family member was unsure whether the WIS person's injury had been sustained on deployment, in training or prior to joining the military or that the injury, or injuries, the WIS person had were both operational and non-operational (Table 1 and online supplementary table 3 ).

Analysis of the data suggested that there was one main, 'umbrella' theme: 'family member involvement' and four subthemes which represented the support needs and experiences of family members of WIS personnel: 'continuity of support', 'proactive signposting and initiating contact', 'psychoeducation and counselling' and 'higher risk groups'.

\section{Family member involvement}

Family members considered themselves to be knowledgeable contributors to the WIS person's recovery process and wanted to offer information to service providers in order to help improve
Table 1 Overview of characteristics of family members and WIS personnel

\begin{tabular}{|c|c|}
\hline & Total \\
\hline \multicolumn{2}{|l|}{ Family member } \\
\hline Spouse/husband & 22 \\
\hline Partner & 3 \\
\hline Mother & 8 \\
\hline Father & 4 \\
\hline \multicolumn{2}{|l|}{ WIS: injury type } \\
\hline Operational & 23 \\
\hline Non-operational & 11 \\
\hline Uncertain & 3 \\
\hline \multicolumn{2}{|l|}{ WIS: engagement type } \\
\hline Regular & 32 \\
\hline Reserve & 5 \\
\hline \multicolumn{2}{|l|}{ WIS: service type } \\
\hline Naval service & 8 \\
\hline Army & 22 \\
\hline RAF & 7 \\
\hline \multicolumn{2}{|l|}{ WIS: rank } \\
\hline Officer & 3 \\
\hline NCO & 18 \\
\hline Other ranks & 16 \\
\hline Total completed and analysed & 37 \\
\hline
\end{tabular}

NCO, non-commissioned officer; RAF, Royal Air Force.

the WIS person's recovery (for all quotations see online supplementary table 4). Additionally, they wanted to learn from service providers about the WIS person's condition and to know how they could best support their recovery.

When family members did not expect to be supported by service providers, since they conceived of the WIS person's recovery as distinct from their own experience, then they were less likely to feel excluded when service providers prioritised the WIS person over the family member. However, when family members felt that they had helpful insights to offer service providers regarding the WIS person's recovery but did not feel welcomed to share these, or when they needed support from service providers but did not feel they were a priority in light of the WIS person's support needs, then this led to family members feeling unsupported.

\section{Continuity of support}

If family members visited the WIS person to whom they were related in a hospital and spoke to medical staff, they frequently received updates on the WIS person's recovery, insights into the condition(s) the WIS person was receiving treatment for and information directing them to relevant support services. However, once the WIS person had been discharged from hospital or rehabilitative centre and returned home, some family members experienced less, if any, support from service providers. In contrast, other family members had found that service providers had always communicated well with them and over long periods.

In order to increase continuity of support for family members, participants said that they would appreciate receiving telephone calls periodically from a professional member of the staff. They suggested that this person would need to be someone who was appropriately trained, thus having an understanding of family life, military culture and the effects of physical/psychological injury. 
Proactive signposting and initiating contact

Family members who had multiple responsibilities (eg, they were parents and/or employed), in addition to providing care for the WIS person, felt that service providers needed to be more proactive in initiating contact to signpost them (signposting is to inform a person who requests support about relevant support services). Family members reported that information regarding support services and the WIS person's medical condition was commonly given to the WIS person to pass on to the relevant family members, thus making the WIS person a 'gatekeeper'. On some occasions, WIS personnel did pass information on to their family, but on others, they did not; thus, the relaying of information could be unreliable. Having a member of the staff who acted as a point of contact and reliable source of information was a recommendation made by participants.

\section{Psychoeducation and counselling}

In some cases, family members felt that the WIS person's behaviour had become difficult to manage, and this could challenge their capacity to cope. Some family members had engaged with counselling or psychoeducation, others had not and yet others had been recommended counselling but had not engaged with it. Frequently, family members recognised that counselling may have positively impacted their capacity to cope. Family members felt that having someone to talk to who understood military culture (rather than a friend, family relation or work colleague) could be particularly supportive, since that person could empathise with their experiences that related to being associated with the military.

\section{Higher risk groups}

Family members of WIS personnel with non-operational injuries felt that they did not receive support or information at the same level as family members of WIS personnel who had operational injuries. This was thought to be because family members of operationally wounded WIS personnel would come into contact with medical or welfare staff in hospital/rehabilitative centres, allowing accurate information to be shared with family members.

Other family members felt reluctant to ask for help due to the stigma surrounding mental health illnesses such as post-traumatic stress disorder (PTSD) and depression. The stigma that was perceived to be associated with mental health illness, potentially as a result of military culture, impeded family members seeking support for themselves since they did not want to 'expose' the WIS person, therefore feeling inhibited by stigma-by-proxy (a term to denote stigma experienced by somebody close to a person with a stigmatised condition).

\section{DISCUSSION}

This study examined the needs and experiences of family members of WIS personnel in the UK. The results suggested that family members of WIS personnel would like for service providers to make contact with family members to signpost them and to be involved in the recovery pathway of the WIS person, under the circumstances where this was necessary and would be of benefit to and respectful of the WIS person. Family members experienced discontinuous support when military staff changed posts or when family members lost contact with hospital staff after the WIS person had returned to the home environment. Family members, particularly those related to WIS personnel with mental health diagnoses, suggested counselling or psychoeducation was helpful in alleviating some of their caregiver burden. Finally, there were certain family members who were at higher risk of not being aware of or not accessing support available to them; namely, those WIS personnel with non-operational injuries and/or mental health illnesses.

\section{Family member involvement}

The findings of this study are consistent with those from a RAND (Research and Development Corporation) report, ${ }^{9}$ investigating military caregiving in the USA, which found that most of the interventions serving military caregivers typically prioritise the ill, injured or wounded SP. Previous research has recommended that service providers consider military ${ }^{11}$ caregivers as 'critical partners' in the execution of treatment plans for WIS personnel and to regard them as providers of valuable emotional support and assistance despite confidentiality laws. ${ }^{12} \mathrm{~A}$ report into traumatic limb loss and the needs of the family also suggested that policy be used to encourage a cultural shift towards family-centred care to ensure that the needs of the family of physically injured WIS personnel are taken into consideration by service providers in the UK. ${ }^{13}$

\section{Continuity of support}

Continuity in the relationship between a single practitioner or a team of practitioners and a patient or family member that extends beyond specific episodes of illness or disease can be an important aspect of support for informal caregivers. ${ }^{14} \mathrm{~A}$ randomised controlled trial found that caregiver burden and distress can be reduced when caregivers receive friendly and socially supportive, one-to-one telephone calls. ${ }^{5}$ Socially supportive one-to-one telephone calls given by a member of the staff, dubbed a 'family care coordinator', who would support the WIS person's family, regardless of whether the WIS person was in a hospital, rehabilitative centre or at home, might serve to decrease their caregiver burden.

\section{Proactive signposting and initiating contact}

The MoD outlines their core policy on 'individual responsibility', ${ }^{7}$ stating that 'each SP is responsible for maintaining the communication links between available support and their families'. Some family members in this study did not realise that there were support services available to them, and this was in part due to the fact that the WIS person, as 'gatekeeper', had not passed on information about support services. Signposting can provide caregivers with adequate and accurate documentation of service providers that they can contact for support to minimise risk to themselves and the patient. ${ }^{5}$ Theoretically, the proposed 'family care coordinator' role could be in a position to signpost family members directly.

\section{Psychoeducation and counselling}

This study found that family members felt they may benefit from psychoeducation and/or counselling in order to educate themselves about the issues the WIS person may experience (eg, mental health diagnoses such as PTSD), how to cope with them, to share experiences with other family members of WIS personnel and to reduce the stigma associated with mental health problems. Although both psychoeducation and counselling services are currently available in the UK, there is a need for families to be aware they exist, for more widespread delivery of such services and for the robust evaluation of these interventions. ${ }^{15}$

\section{Higher risk groups}

Recovery pathways for operationally injured and non-operationally injured personnel are supposed to be the same, ${ }^{7}$ however, 
support received by family members of WIS personnel with operational injuries was perceived by family members to be better than the support received by family members of WIS personnel with non-operational injuries. In order to ensure that support for family members of WIS personnel with different origins of injury is the same, further, robust and empirical investigation is warranted.

The results of the current study suggest that families of WIS personnel with mental health diagnoses may face more barriers to help-seeking than their operationally injured counterparts due to stigma-by-proxy. This finding is cited in the literature, where it has been suggested that family members may fear adverse consequences (eg, negative occupational and social outcomes) of seeking help. ${ }^{16}$ Family members of service personnel with mental health diagnoses may be more likely to seek help if they are offered systematic reassurance from support service providers regarding their feared, adverse consequences of help-seeking. ${ }^{16}$

\section{Limitations}

A low response rate meant that our sample was not representative of the wider military population. There were significantly more WIS personnel from the Army who were regulars than from the Naval Service, RAF or reserve forces; male family members of WIS personnel were also under-represented. The sample was made up of family members of both wounded and sick personnel, rather than of family members of WIS personnel with operational or non-operational injuries/illnesses. Using a different recruitment strategy that did not rely on accessing family members via the WIS personnel themselves might help to achieve a more diverse sample population; furthermore, it may be beneficial to focus on the experiences of family members of either wounded or sick service personnel in order to explore the potentially different support needs of these groups.

In interview, participants were not routinely asked whether the SP to whom they were related had been medically discharged or not; therefore, the authors were unable to consistently report whether the WIS person in question was returning to their unit or on a discharge pathway. This limitation does not allow the reader to contextualise the different possible expectations that may be placed on the family according to these circumstances. Despite these limitations, this study was the first of its kind to identify key recommendations for the MoD with regard to the support needs of family members of WIS personnel in the UK.

\section{Recommendations}

We recommend robust, empirical investigation is carried out to examine whether systematic differences are indeed occurring between support service provision for family members of WIS personnel with operational or non-operational injuries; furthermore, if necessary, what recommendations can be made in order to rectify these.

Family members of WIS service personnel in the UK may benefit from support provided by 'family care coordinators', a role that does not exist currently within service provision. 'Family care coordinators' could decrease the amount of responsibility currently apportioned to service personnel ${ }^{7}$ by creating a professional role which would aim to enhance levels of effective signposting, increase family involvement and provide continuous and consistent care in the WIS person's recovery pathway (where this did not compromise the wishes or medical confidentiality of the WIS person). We suggest that this proposed role be further discussed with key stakeholders to discern its relevance and suitability, then piloted to discern its feasibility and if implemented, then robustly evaluated to determine its effectiveness.

\section{CONCLUSIONS}

The findings of this study suggest that family members of WIS personnel would value proactive, direct and sustained communication from support service providers (where this does not compromise the wishes or medical confidentiality of the WIS person); thus, somewhat decentralising the role of the WIS person in support service provision for families. Family members of WIS personnel with non-operational injuries indicate that they would appreciate being made equally aware of, and able to access, support services as their operationally injured counterparts. Robust, empirical investigations are necessary to examine whether systematic differences are indeed occurring between these two groups. Finally, family members of WIS service personnel in the UK may benefit from support provided by 'family care coordinators', a role that may go some way in providing continuous support and increased family involvement from the moment of injury until family members opt out of this service.

Contributors NTF was the principal investigator of the study. MK was the study coordinator. AV and GT carried out participant interviews and data analysis. SAMS was involved in advising on the study interview schedule, data collection and writing of the manuscript. AV drafted the manuscript. All authors read and approved the final manuscript.

Funding TIN 2.025 (B)

Competing interests King's Centre for Military Health Research receives some funding from the MoD.

Patient consent Obtained.

Ethics approval MODREC 502MOD13.

Provenance and peer review Not commissioned; externally peer reviewed.

Data sharing statement Some data may be accessible upon request.

(c) Article author(s) (or their employer(s) unless otherwise stated in the text of the article) 2017. All rights reserved. No commercial use is permitted unless otherwise expressly granted.

\section{REFERENCES}

1 House of Commons Public Accounts Committee. Ministry of defence: support to high intensity operations. London: The Stationery Office Limited; 2009, Report No: HC 895. (accessed 13 Oct 2009).

2 MoD. MoD health and safety statistics annual report 2007/08 - 2013/14. Bristol (UK): Minstry of Defence; 2014, Report No: 2007/08 - 2013/14. (accessed 31 Oct 2014)

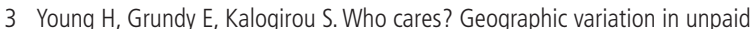
caregiving in England and Wales: evidence from the 2001 census. Popul Trends 2005:23-34

4 Campbell CL, McCoy S, Hoffman N, et al. Decreasing role strain for caregivers of veterans with dependence in performing activities of daily living. Health Soc Work 2014:39:55-62.

5 Reinhard S, Given B, Huhtala Petlick N, et al. Supporting Family Caregivers in Providing Care. Hughes RG, Patient safety and quality: an evidence-based handbook for nurses. Rockville (MD): Agency for Healthcare Research and Quality, 2008:341-404

6 MoD. The armed forces covenant. UK: Ministry of Defence, 2011:1-16.

7 MoD. Tri Service Operational and Non Operational Welfare Policy. In: Directorate of Service Personnel Policy: Ministry of Defence, 2014:1-205.

8 House of Commons Defence Committee. The armed forces covenant in action? part 1: military casualties: seventh report of sessions 2010-2012. London: The Stationery Office Limited, 2011.

9 Ramchand R, Tanielian T, Fisher M, et al. Hidden heroes: America's military caregivers. Santa Monica, California: RAND Corporation, 2014

10 Braun V, Clarke V. Using thematic analysis in psychology. Qual Res Psychol 2006;3:77-101.

11 Hall C, Sigford B, Sayer N. Practice changes associated with the Department of Veterans Affairs' Family Care Collaborative. J Gen Intern Med 2010;25 Suppl 1:18-26. 
12 Sherman MD. The SAFE Program: a family psychoeducational curriculum developed in a veterans affairs medical center. Prof Psychol 2003;34:42-8.

13 Fossey M, Hacker Hughes J. Blesma, The Limbless Veterans. Traumatic limb loss and the needs of the family. 2014

14 Haggerty IL, Reid RJ, Freeman GK, et al. Continuity of care: a multidisciplinary review. BMJ 2003:327:1219-21.
15 Verey A, Keeling M, Thandi G, et al. UK support services for families of wounded, injured or sick service personnel: the need for evaluation. J R Army Med Corps 2016;162:324-5.

16 Jones N, et al. Mental health, stigmatising beliefs, barriers to care and helpseeking in a non-deployed sample of UK army personnel. J Psychol Psychother 2013;03. 